\title{
Designing a Hardware Platform for Training Operators of Critical Infrastructures
}

\author{
Neamţu CĂLIN \\ Aptus Software, Bucharest, Romania \\ Technical University of Cluj-Napoca, Cluj-Napoca, Romania \\ calin.neamtu@muri.utcluj.ro \\ Camelia ACHELARIȚEI \\ Avitech Co, Bucahrest, Romania \\ camelia.achelaritei@avitech.ro \\ Ion ANGHEL \\ Police Academy, Fire Officers Faculty, Bucharest, Romania \\ ion_anghel2003@yahoo.com \\ Ştefan BODI \\ Technical University of Cluj-Napoca, Cluj-Napoca, Romania \\ stefan.bodi@muri.utcluj.ro
}

\begin{abstract}
Critical infrastructures could endanger the trainees' lives and/or the environment. The nature of these infrastructures often does not allow staff training in real situations. This paper presents the processes involved in designing a virtual reality platform (hardware and software) to provide a safe training environment, which is believed to be able to better support in the training of staff involved in critical infrastructures. The design of this training platform includes the considerations and adoption of an innovation algorithm based on the TRIZ method. The paper addresses the training process of personnel who operate offshore drilling facilities. The platform is in the physical development stage within the CREVIS project and it will allow personnel training from critical infrastructures in a mixed environment: real / virtual.
\end{abstract}

Keywords: training using virtual reality, virtual reality cave system, critical infrastructure training

\section{INTRODUCTION}

Critical infrastructures are those facilities that have an important role in ensuring the security of systems in their functioning and in the flow of economic, social, political, informational 
and military processes. According to (Commission of the European communities, 2008) critical infrastructures in Europe include: highway systems, ports, etc., that provide the functioning fundamentals for an economy, physical networks used to connect computers and users and power, water and gas supply systems. These systems cannot be used effectively in the training of personnel without seriously disrupting the normal activities of their operation. Thus, staff training is done mostly in a theoretical way and in some cases on replicas of real systems, in which case the costs are high. Using virtual reality for training operators that work in critical infrastructures is already put into practice by several prestigious institutions such as: New York City Office of Emergency Management (Advanced Disaster Management Simulator - ADMS, the system is concentrated on the simulation of command elements and allows the trainee to navigate within the virtual city with the help of a joystick (Hsu EB, 2013)) or the Los Angeles Police Department (Hydra simulation system for training command officers for disaster category incidents (Pittman, 2010)). In the field of training operators for offshore drilling platforms there are a number of training providers who already use complex virtual reality systems and simulation software. Among these we mention: Worldviz (Wordviz, 2015), that uses a CAVE-like projection system with 5 walls, built by Christie Digital; CM Labs, that uses the Vortex system (Figure 1 - left (CM Labs, 2015)); and Ari Marine, that includes also a virtual bridge with a $360^{\circ}$ projection (Figure 1 - right (Ari Marine, 2015)).
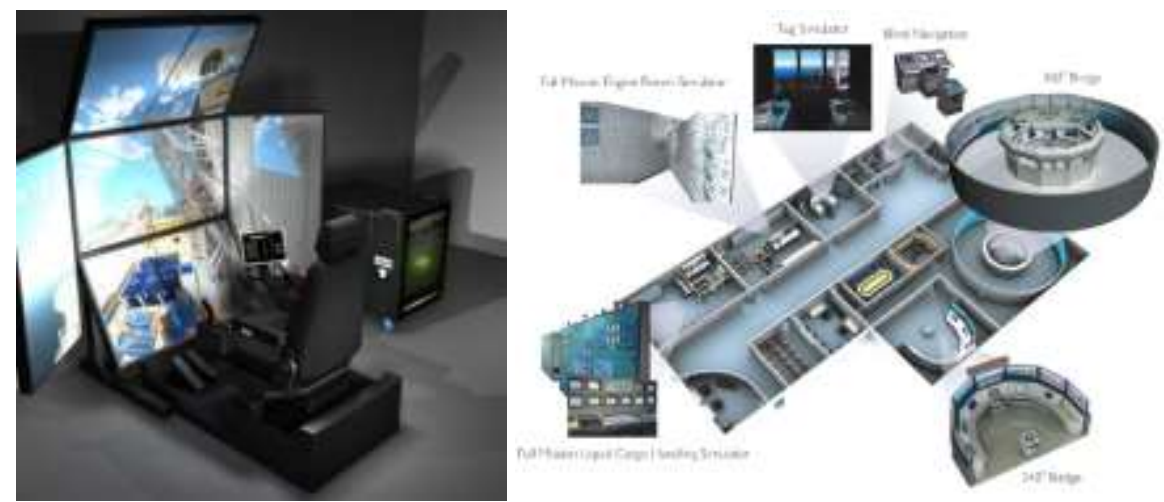

Figure 1: Vortex Master left (CM Labs, 2015), and Ari Marine (Ari Marine, 2015)

\section{WORK METHODOLOGY}

The platform development was done using the methodology presented in Figure 2, based on a QFD - TRIZ combination and with the help of support instruments specific to competitive development.

The methodology (Figure 2) starts with the Need Analysis, where using instruments like Brainstorming, Affinity Diagram and Tree Diagram requirements are identified, which refer to the training system. The two diagram-type instruments are used to group and identify stakeholder requirements related to the training process: the employer of the trainee, the trainee, the trainer and the training supplier. 
In the second, the Benchmark Needs stage, the main competitors are identified, the needs are prioritized using the AHP (Analytical Hierarchy Process) method, the Main Sales Points are established and the Competitor's Performance Levels is determined. Based on the information gathered until this point the training system's Target Performance Level will be established.

The third phase of the work methodology is the Analyze CTQs (Critical-to-Quality), in which classic analysis methods (Brainstorming, Affinity Diagram and Tree Diagram) are used to identify the CTQ elements. These elements will constitute as inputs for the QFD Matrix, the fourth stage. After completing the QFD matrix, the correlations between CTQs, the strength of the relationships and the optimization directions will be identified for each requirement related characteristic.

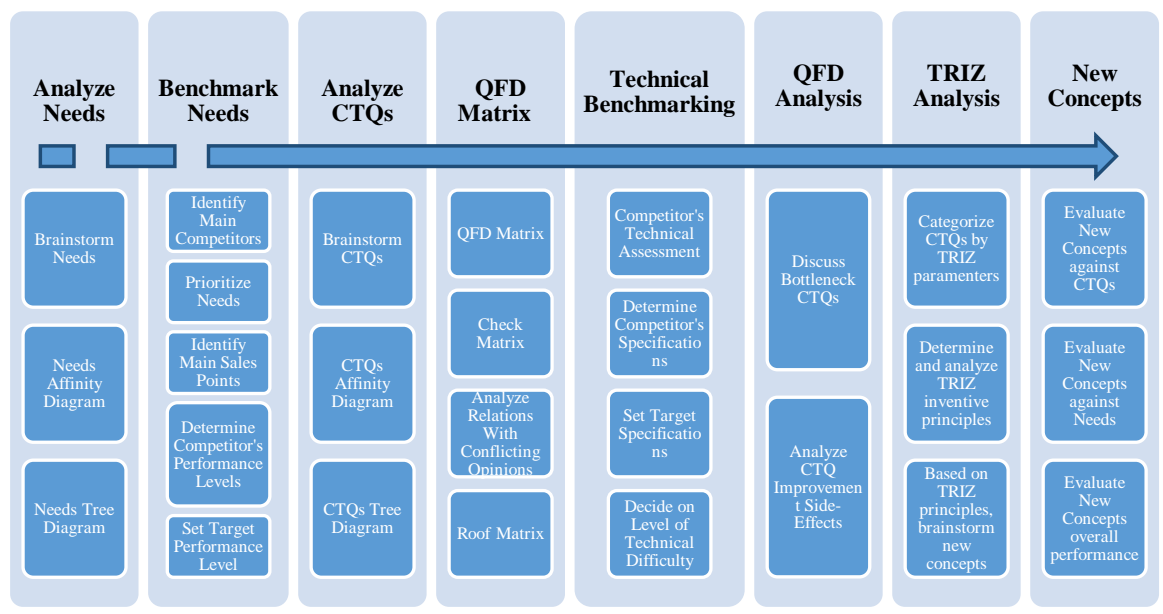

Figure 2: Work methodology

In the next stage (the fifth), called Technical Benchmarking a technical evaluation will be carried out following which the important technical specifications are determined. These are grouped into a targeted technical specification set for the training system, which are later analyzed from the difficulty-in-implementation point of view.

In the sixth stage (QFD Analysis) Bottlenecks and CTQ Improvement Side-Effects are analyzed (technical impact analysis) and the obtained CTQs represent inputs for the TRIZ method.

The seventh phase implies the use of the TRIZ Analysis for generating innovative technical solutions. Thus, each CTQ is equated with a TRIZ method parameter, the main conflicts and the applicable inventive principles are determined and then generic solutions are searched, based on previously known solutions. In the final step of the TRIZ method the generic solutions are interpreted and technical solutions are established in the form of new concepts. 
In the final point of the work methodology (New Concepts) the concepts are evaluated in comparison with the Needs and the CTQs for determining the extent to which these fulfill the initial needs and requirements.

\section{RESULTS}

For efficiently managing the algorithm implementation the Qualica QFD software solution was used as support instrument for deploying the TRIZ, QFD and AHP methods. In the Need Analysis stage a set of 44 requirements was identified for the training system. Using the Affinity Diagram these were grouped and concentrated into 21, which were finally considered as inputs for the Benchmark Needs stage. The main identified competitors are: Ari Simulation, Oiltec Solution, Cubic, QinetiQ, Ksim, Ploaris and Plexsys. The hierarchy of the first five needs resulted from the Benchmark Needs is the following:

1. The ability to create dynamic scenarios

2. To allow the creation of test scenarios

3. Allow the introduction of random elements in scenarios

4. Smart equipment can use for interaction

5. The ability to create or import 3D models

The graphic representation of the entire Benchmark Needs is presented in Figure 3.

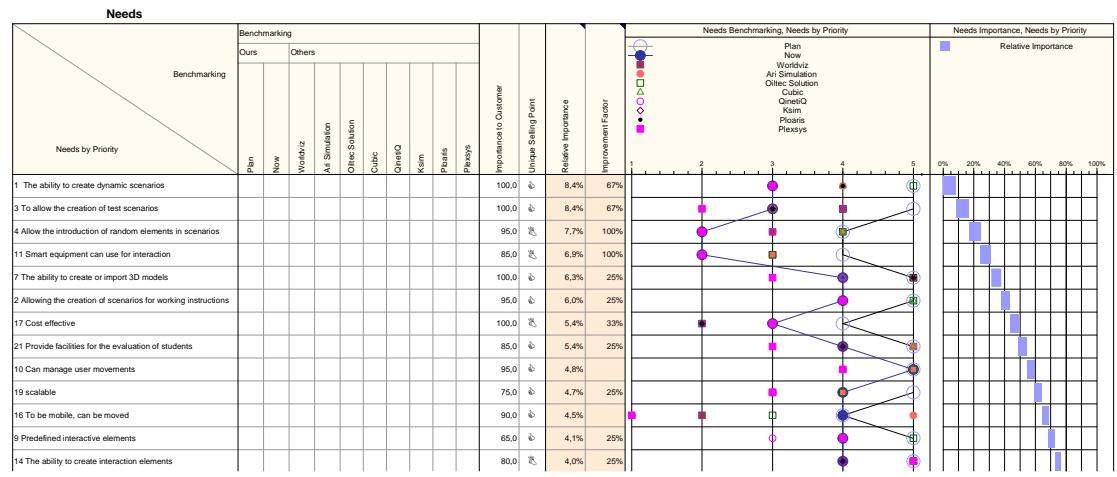

Figure 3: Benchmark Needs results

The prioritization matrix (Figure 4) offers an overview of the correlations between the needs and CTQs and allows the realization of a rigorous implementation plan, based on the importance and influence of the CTQs upon the needs. 


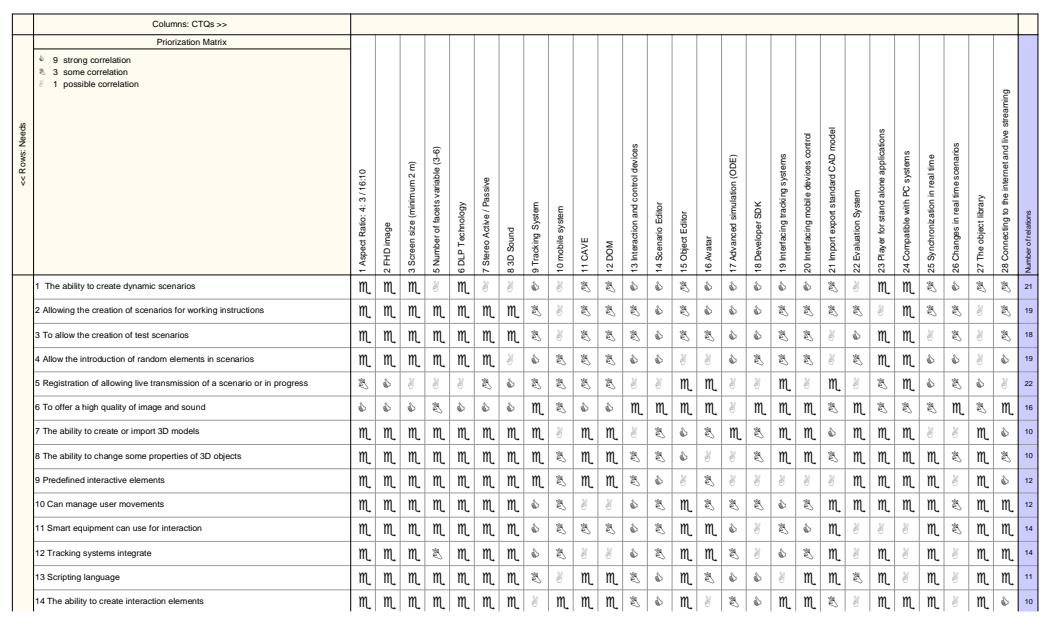

Figure 4: Prioritization Matrix (extract)

In the Technical Benchmarking stage (Figure 5) the following bottlenecks were identified for which solutions have to be found in the innovation phase:

- Tracking System - the necessity of integrating one or more tracking systems (for example, tracking system for the entire body and tracking system only for the hands)

- Scenario Editor - the possibility of creating independent or correlated scenarios

- Advanced simulation - simulating natural phenomena in a realistic way

- Changes in real time scenarios - dynamic modification of a predefined scenario

- Connecting to the internet and live streaming - transmitting and recording a training event

After establishing and prioritizing the CTQs in the QFD stage, within the first step of the TRIZ method they are attributed a TRIZ parameter. The attribution is done based on the similarities between the elements; the association is presented in Table 1.

After attributing the TRIZ parameter the calculation of the contradiction matrix follows; this is done automatically in the Qualica QFD software. The first step in using the above mentioned matrix consists in selecting a parameter that is to be improved (rows). The second step consists in identifying the intersection of the selected parameter with one or more parameters that are in conflict (columns). 


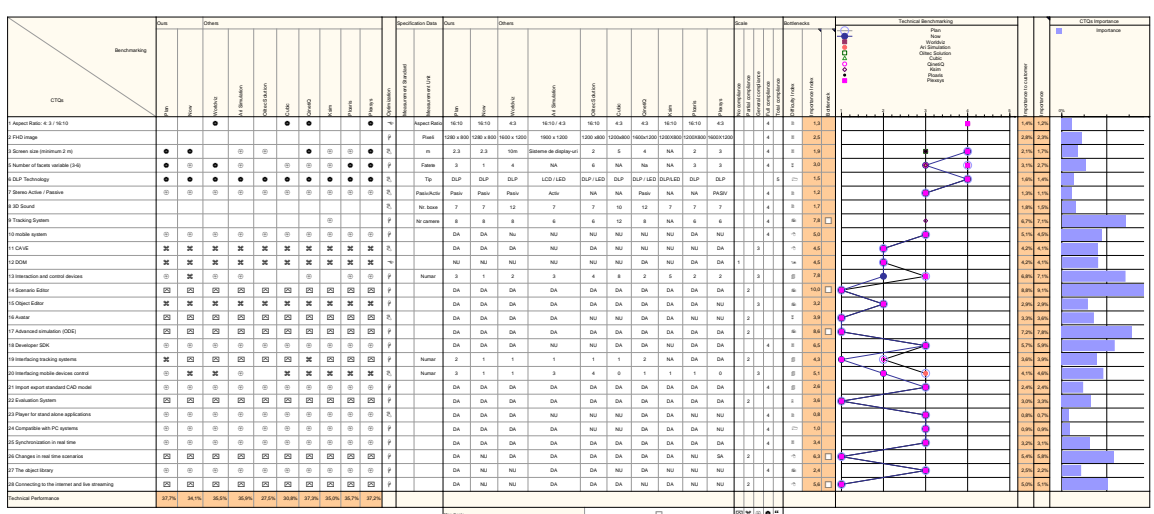

Figure 5: Technical Benchmarking

Table 1: Corespondence between CTQ and TRIZ parameter (Extract)

\begin{tabular}{|l|l|}
\hline Aspect Ratio: 4: 3 / 4:10 p.m. & The brilliance / Clarity \\
\hline FHD image & Strength \\
\hline Screen size (minimum 2 m) & The ease of being manufactured \\
\hline Number of facets variable (3-6) & The complexity of device / your system's \\
\hline DLP Technology & The complexity of device / your system's \\
\hline Stereo Active / Passive & The brilliance / Clarity \\
\hline 3D Sound & Power \\
\hline Tracking System & Speed \\
\hline mobile system & Volume object in motion \\
\hline CAVE & The complexity of device / your system's \\
\hline DOM & The complexity of device / your system's \\
\hline
\end{tabular}

Using Qualica QFD the number of conflicts and solutions are automatically identified with the help of the tables specific to the method. The extracted inventive principles do not represent the solution or solutions for the problem.

They simply indicate a few directions where specialists must look for innovative solutions that can resolve the conflict without compromise. For example, in the case of the Aspect Ratio 4:3 / 16:10 CTQ 23 conflicts were identified. This CTQ recorded conflicts with: Screen Size (min. 2 m), Number of facets variable (3-6), Tracking System, Interaction and control devices, Interfacing mobile devices control, Synchronization in real time, etc.

For the conflict between Aspect Ration 4:3 / 16:10 and Screen size (min. 2 m) the TRIZ Manufacturability parameter was attributed and the following inventive principles were found (see Table 2): Periodic action, Copying, Replacement of a mechanical system and Transformation of the physical and chemical states of an object. 


\section{Table 2: Details about Inventive principle.}

\begin{tabular}{|l|l|}
\hline Periodic action & $\begin{array}{l}\text { Replace a continuous action with a periodic (pulsed) } \\
\text { one. If an action is already periodic, change its } \\
\text { frequency. Use pulsed between impulses to provide } \\
\text { additional action }\end{array}$ \\
\hline Copying & $\begin{array}{l}\text { Use a simple and inexpensive copy instead of an } \\
\text { object that is complex, expensive, fragile or } \\
\text { inconvenient to operate. Replace an object by its } \\
\text { optical copy or image. A scale can be used to reduce } \\
\text { or enlarge the image. If visible optical copies are } \\
\text { used, replace them with infrared or ultraviolet copies }\end{array}$ \\
\hline $\begin{array}{l}\text { Replacement of a } \\
\text { mechanical system }\end{array}$ & $\begin{array}{l}\text { Replace a mechanical system by an optical, } \\
\text { acoustical or olfactory (odor) system. Use an } \\
\text { electrical, magnetic or electromagnetic field for } \\
\text { interaction with the object. Replace fields: } \\
\text { Stationary fields with moving fields; Fixed fields } \\
\text { with those which change in time; Random fields } \\
\text { with structured fields. Use a field in conjunction } \\
\text { with ferromagnetic particles }\end{array}$ \\
\hline $\begin{array}{l}\text { Transformation of the } \\
\text { physical and chemical } \\
\text { states of an object }\end{array}$ & $\begin{array}{l}\text { Change an object's aggregate state, density } \\
\text { distribution, and degree of flexibility, temperature }\end{array}$ \\
\hline
\end{tabular}

One of the new developed concepts was based on the following elements: Use a simple and inexpensive copy instead of an object that is complex + Replace a continuous action with a periodic (pulsed) one. If an action is already periodic, change its frequency - the use of a single projector with a minimum frequency of $120 \mathrm{~Hz}$ (instead of two projectors) as a source for the stereoscopic image.

After using the TRIZ method a set of new concepts was identified, applicable to system development for training operators of critical infrastructures. In the final step of this methodology they were analyzed from the identified needs and CTQs point of view, such that by putting them into practice the development of the desired system will be ensured. In Figure 6 the result of this analysis is shown.

After analyzing the concepts presented above these have been grouped into three distinct systems: the software simulation and 3D projection system, the hardware projection and interaction system and the online database with predefined 3D objects and scenarios.

After defining the three systems a market research was carried out for identifying a customizable software solution, that can be used in such a way that the software development part will not start from scratch. This study answers to the identified need of integrating an existing technical solution. After identifying this solution (Eon Studio) the focus was shifted to the design of the projection system (Figure 7). This is composed out of a removable metallic frame (1) shaped as a cube, upon which 3, 4, 5 or 6 projection screens (2) are mounted. 


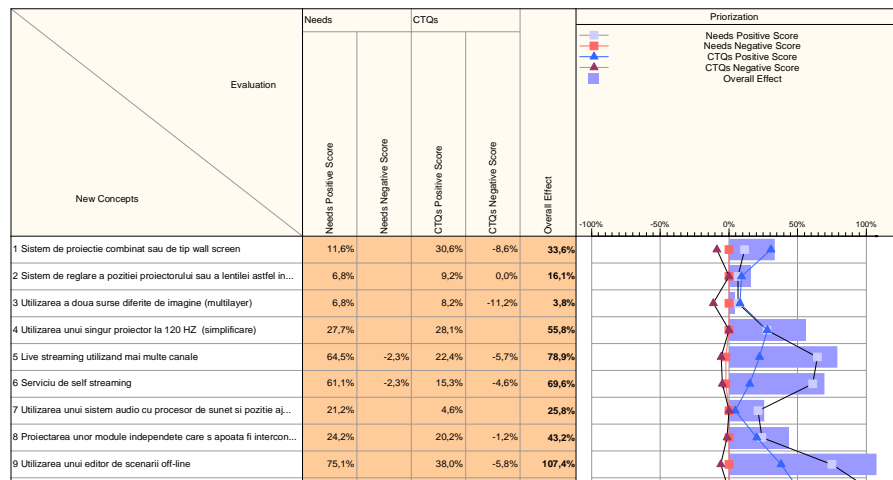

Figure 6: New concepts evaluation (extract)

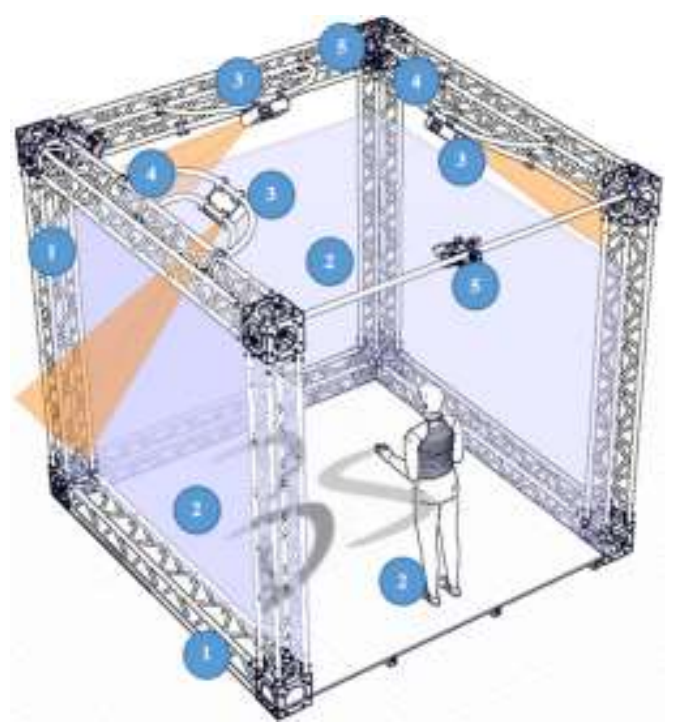

Figure 7: 3D model of the training system

On each side of the cube a 3D projector (3) is mounted that will project on each screen a part of the $3 \mathrm{D}$ environment, used in the software simulation of a scenario. The projectors can be fixed on the support beams of the cube with the help of adjustable systems (4), inside or out, depending on the number of used screens. On the metallic structure the sound system and the cameras of the tracking system (5) are mounted.

Because a requirement was identified related to the design of reconfigurable modules that can be connected to the training system for allowing the trainee to enter commands in the simulation using real actuating elements familiar to him from his workplace, a multifunctional panel was designed. This is mounted within the projection system and allows direct interaction with the virtual environment through the command elements placed on it. Other than these, the user can interact with a range of virtual command instruments placed 
on one of the screens used for the 3D projection. The virtual instruments are actuated by gestures recorded by the tracking system.

\section{CONCLUSION}

Using the presented algorithm led to the detailed design of a complex training system, that is in the physical development stage. Every electronic component of the projection system is standardized; the metallic support structure of the projectors and of the other systems (sound tracking, etc.) is customized and was built out of standardized aluminum profiles. The approximate cost of the system is 350,000 EUR for the 4-wall version.

\section{ACKNOWLEDGEMENT}

This paper has benefited from the support of the project "Achievement of an innovative platform based on virtual reality applications for the personnel's safety and protection in critical infrastructures - CREVIS”, Project Nr. P019002 / 1775 SIMIS Code: 50433, beneficiary Aptus Software SRL - Bucureşti. The project is part of the CREVIS pole of competitiveness financed through the Sectorial Operational Program "Increase of Economic Competitiveness 2007- 2013" by the European Regional Development Fund. This publication reflects the views only of the authors, and the Commission cannot be held responsible for any use which may be made of the information contained therein.

\section{REFERENCES}

COMMISSION OF THE EUROPEAN COMMUNITIES, COUNCIL DIRECTIVE 2008/114/EC of 8 December 2008, On the identification and designation of European critical infrastructures and the assessment of the need to improve their protection, Retrieved March 15, 2015 from http://europa.eu.int/eurlex/lex/LexUriServ/site/fr/com/2004/com2004 0702fr01.pdf

Hsu EB, Li Y, Bayram JD, Levinson D, Yang S, Monahan C. State of Virtual Reality Based Disaster Preparedness and Response Training. PLOS Currents Disasters. 2013 Apr 24. Edition 1. doi:

10.1371/currents.dis.1ea2b2e71237d5337fa53982a38b2aff

Elaine Pittman (2010). Los Angeles Police Department Hydra System Promotes Simulation Training for Command-Level Officers, Government Technology Retrieved March 15, 2015 from http://www.govtech.com/public-safety/Los-Angeles-Police-HydraSimulation-Training.html

Wordviz, (2015). Be There with Immersive 3D Interaction, Retrieved March 15, 2015 from http://www.worldviz.com/industries/training

CM Labs (2015), Trainning System for energy system, Retrieved May 10, 2015 from http://www.cm-labs.com/energy-offshore

Ari Marine, Drilll simulator, Retrieved June 15, 2015 from: http://www.arisimulation.com/products/drilling-simulators 